\title{
Species Identification and Clarithromycin Susceptibility Testing of 278 Clinical Nontuberculosis Mycobacteria Isolates
}

\author{
Wenjuan Nie, ${ }^{1}$ Hongfei Duan, ${ }^{1}$ Hairong Huang, ${ }^{2}$ Yu Lu, ${ }^{3}$ and Naihui Chu ${ }^{1}$ \\ ${ }^{1}$ Department of Tuberculosis, Beijing Chest Hospital, Capital Medical University, Tongzhou District, Beijing 101149, China \\ ${ }^{2}$ Reference Lab, Beijing Chest Hospital, Capital Medical University, Tongzhou District, Beijing 101149, China \\ ${ }^{3}$ Department of Pharmacology, Beijing Chest Hospital, Capital Medical University, Tongzhou District, Beijing 101149, China
}

Correspondence should be addressed to Hairong Huang; hairong.huangcn@gmail.com and Naihui Chu; chunaihui1994@sina.com

Received 22 July 2014; Accepted 20 December 2014

Academic Editor: Parissa Farnia

Copyright (C) 2015 Wenjuan Nie et al. This is an open access article distributed under the Creative Commons Attribution License, which permits unrestricted use, distribution, and reproduction in any medium, provided the original work is properly cited.

\begin{abstract}
Purpose of this paper is to analyze different species' proportion of nontuberculosis mycobacteria (NTM) and susceptibility to clarithromycin of different species. 278 clinical NTM isolates were identified into species by using $16 \mathrm{~S}$ rRNA, $r p o B$ and $h s p 65$. Then clarithromycin susceptibility testing against different species was done separately, using microplate Alamar Blue assay. Finally, resistance isolates' erm(41) of $M$. abscessus were sequenced in order to analyze mechanisms for clarithromycin resistant. In this test, 131 isolates (47\%) belonged to M. avium complex (MAC), and 70 isolates (25\%) belonged to M. abscessus. Nearly all the M. abscessus subsp. abscessus resistant to clarithromycin had T28 in erm(41). However, all the M. abscessus subsp. abscessus susceptible to clarithromycin had C28 in erm(41). In this study, we find that MAC was the most common pathogens of NTM, and the second one was $M$. abscessus. However, M. chelonei, M. fuerth, and M. gordon were rare. Clarithromycin had a good inhibition activity against all the NTM species except M. abscessus subsp. abscessus. The erm(41) genotype is of high relevance to clarithromycin resistance.
\end{abstract}

\section{Introduction}

Nontuberculosis mycobacteria (NTM) are globally recognized as pathogens especially in immunocompromised population including HIV/AIDS (Human Immunodeficiency Virus/Acquired Immunodeficiency Syndrome) patients. In addition to causing infections in immune-compromised patients, this group of organisms has been reported to be increasingly recognized as pathogens from immunocompetent individuals as well [1]. Different species have different susceptibility to antibiotics, so it is important to identify NTM into different species in order to make the respective therapy plan. The American Thoracic Society (ATS) and the Infectious Diseases Society of America (IDSA) recommended treatment for NTM pulmonary and disseminated disease is a macrolide-containing, multidrug regimen. And clarithromycin, a macrolide drug, is described as the key therapy for NTM diseases [2]. Clarithromycin forms the cornerstone of NTM treatment and is the only drug recommended by the Clinical and Laboratory Standards Institute
(CLSI) for drug susceptibility testing (DST) [3]. However, antibiotic treatment of pulmonary $M$. abscessus infections remains problematic. Infections due to the $M$. abscessus group are difficult to treat because these mycobacteria are intrinsically resistant not only to the classical antituberculous drugs but also to most of the antibiotics that are currently available. Clarithromycin had been the drug of choice for $M$. abscessus infections for the past 20 years before a resistance gene, erm(41), was described in M. abscessus. The discovery of a novel inducible erm gene, erm(41), provides an explanation for the lack of efficacy for macrolides against M. abscessus infections [4]. A polymorphism in erm(41) ( $\mathrm{T}$ or $\mathrm{C}$ ) was described at position 28 and its association with the susceptibility was examined. Our objective was to identify NTM isolates into different species and to do the DST separately and then to seek the presence of erm(41) in a large collection of clinical strains precisely identified as $M$. abscessus and finally to correlate these results with clarithromycin susceptibility. 


\section{Materials and Methods}

2.1. Species Identification. All of the 278 clinical strains in Reference Lab of Beijing Chest Hospital were isolated from sputum samples of patients, which were previously identified as NTM strain using growth experiments of pnitrobenzoic acid (PNB) by Reference Lab of Beijing Chest Hospital. All the strains were subcultured on the LowensteinJensen medium at $37^{\circ} \mathrm{C}$ to observe colony morphology. The incubation time ranged from 4 to 28 days. Then colonies were used for species identification based on 16S rRNA sequence analysis. Preparation of the PCR reaction mixture and amplification were done as described previously $[5,6]$. PCR products were purified and sequenced by the BGI corporation, using forward and reverse primers. Both strands were sequenced as a crosscheck. Species identification of these strains was accomplished by the sequencing of $16 \mathrm{~S}$ rRNA, using BLAST search to measure the similarities.

\subsection{Drug Susceptibility Testing. We performed DST of NTM} isolates by broth microdilution using 96-well plates, which is a reference method as recommended by CLSI [3]. DST of these NTM isolates was done as described previously [6]. To M. abscessus, plates were submitted to an extended incubation of 14 days incubation at $30^{\circ} \mathrm{C}$. However, to other NTM isolates, the incubation time ranged from 3 to 10 days. A color change from blue to pink indicates bacterial growth. MIC was defined as the lowest concentration of the drug that showed no color change, which was the lowest concentration of drug capable of inhibiting the visible growth of tested isolates. $\mathrm{MIC}_{50}$ and $\mathrm{MIC}_{90}$ values were defined as drug concentrations, which inhibit $50 \%$ and $90 \%$ of isolates, respectively. Susceptibility was evaluated according to CLSI breakpoint recommendations [3]. The type strains of American Type Culture Collection (ATCC) were used for comparison.

2.3. Clarithromycin Resistant Mechanism. Crude DNA preparations suitable for PCR were obtained by heating suspensions of mycobacteria in Tris-EDTA buffer at $100^{\circ} \mathrm{C}$ for 15 to $20 \mathrm{~min}$. Debris was removed from the preparations by centrifugation at $16,000 \mathrm{rpm}$ for $3 \mathrm{~min}$. The basic $40 \mu \mathrm{L}$ PCR mixture comprised $2 \mu \mathrm{L}$ DNA, $2 \mu \mathrm{L}$ DNA polymerase, and $1 \mu \mathrm{L}$ each primer and finally was complete to $40 \mu \mathrm{L}$ by double distilled water. Reactions were run for $3 \mathrm{~min}$ at $94^{\circ} \mathrm{C}$, followed by 30 cycles at $94^{\circ} \mathrm{C}$ for $1 \mathrm{~min}, 61^{\circ} \mathrm{C}$ for $1 \mathrm{~min}$, and $72^{\circ} \mathrm{C}$ for $1 \mathrm{~min}$. For cloning of erm(41), gene segments were amplified with primers 5-ACG TTG GAT CCG AGC GCC GTC ACA AGA TGC ACA-3 and 5-GCG AGA AGC TTG ACT TCC CCG CAC CGA TTC CAC-3. The resulting amplification products were purified and sequenced by the BGI corporation, using forward and reverse primers. Both strands were sequenced as a crosscheck. The sequence results were compared with M. abscessus standard strains.

\section{Result}

3.1. Species Identification. Of all the 278 NTM clinical strains (Table 1), 100 (36\%) isolates belonged to rapidly growing Mycobacterium (RGM), and 178 (64\%) isolates belonged to
TABLE 1: The result of NTM species identification.

\begin{tabular}{lcc}
\hline & \multicolumn{1}{c}{ Species } & Number (\%) of strains \\
\hline \multirow{2}{*}{ RGM } & M. abscessus & $70(25 \%)$ \\
$n=100(36 \%)$ & M. fortuitum & $23(8 \%)$ \\
& M. chelonei & $5(2 \%)$ \\
& M. fuerth & $2(1 \%)$ \\
\hline \multirow{2}{*}{ SGM } & MAC & $131(47 \%)$ \\
$n=178(64 \%)$ & M. kansasii & $34(12 \%)$ \\
& M. gordon & $13(5 \%)$ \\
\hline Total $n=278(100 \%)$ & NTM & $278(100 \%)$ \\
\hline
\end{tabular}

slowly growing Mycobacterium (SGM). Among these species, 70 isolates (25\%) belonged to M. abscessus, 23 isolates (8\%) belonged to $M$. fortuitum, 5 isolates (2\%) belonged to $M$. chelonei, 2 isolates (1\%) belonged to M. fuerth, 131 isolates (47\%) belonged to $M$. avium complex (MAC), 34 isolates (12\%) belonged to M. kansasii, and 13 isolates (5\%) belonged to M. gordon. Of the $70 \mathrm{M}$. abscessus strains, according to $r p o B$ and $h s p 65,45$ strains (64\%) belonged to M. abscessus subsp. abscessus, and 25 strains (36\%) belonged to M. abscessus subsp. massiliense.

All the $70 \mathrm{M}$. abscessus isolates were divided into two subspecies, M. abscessus subsp. abscessus and M. abscessus subsp. massiliense (Table 2). There was no M. abscessus subsp. bolletii strain observed. There were two ways to identify subspecies: sequencing $r p o B$ PCR-restriction fragments and sequencing $h s p 65$ PCR-restriction fragments. Results of these two methods were the same (M. abscessus subsp. abscessus, $64 \%$; M. abscessus subsp. massiliense, $36 \%$ ), which means that all the M. abscessus subsp. abscessus and M. abscessus subsp. massiliense strains divided by $r p o B$ sequence were all verified by $h s p 65$ sequence. There was no discordant result between $r p o B$ and $h s p 65$ sequences analysis. The $r p o B$ similarity of $M$. abscessus subsp. abscessus to the reference sequences was $98 \%$ to $100 \%$, and the $h s p 65$ similarity was $98 \%$ to $100 \%$. The $r p o B$ similarity of $M$. abscessus subsp. massiliense to the reference sequences was relatively low, $97 \%$ to $100 \%$, and the $h s p 65$ similarity was $98 \%$ to $100 \%$.

3.2. Drug Susceptibility Testing. The breakpoint, MIC range, $\mathrm{MIC}_{50}, \mathrm{MIC}_{90}$, and proportions of susceptible, moderately susceptible, and resistant strains of each drug against NTM are shown in Table 3, respectively. As RGM, M. fortuitum should be incubated for 3 days (CLSI recommends), and then the MIC value is read. In Table 3, it shows that most of $M$. fortuitum (92\%) strains were susceptible to clarithromycin, and the resistant proportion was low (4\%). Although $M$. abscessus belong to RGM, the new CLSI guideline extended the incubation time to 14 days, which is the shortest time CLSI recommends for final reading. In this test, $M$. abscessus was divided into different subspecies, M. abscessus subsp. abscessus and M. abscessus subsp. massiliense, and the DST was done separately. It appeared that most of the M. abscessus subsp. massiliense strains are susceptible to clarithromycin, whereas susceptibility was less clear for $M$. abscessus subsp. abscessus, with most strains being resistant 
TABLE 2: Each subspecies' proportion identified by using rpoB and $h s p 65$.

\begin{tabular}{lccc}
\hline Sequence analysis & Number (\%) of M. abscessus subsp. abscessus & Number (\%) of M. abscessus subsp. massiliense & Total number (\%) \\
\hline rpoB & $45(64 \%)$ & $25(36 \%)$ & $70(100 \%)$ \\
hsp65 & $45(64 \%)$ & $25(36 \%)$ & $70(100 \%)$ \\
\hline
\end{tabular}

TABLE 3: Clarithromycin susceptibility testing result of different species.

\begin{tabular}{|c|c|c|c|c|c|c|c|c|c|c|}
\hline Subspecies & $\begin{array}{l}\text { Incubation } \\
\text { days }\end{array}$ & $\begin{array}{l}\text { Susceptible } \\
\text { breakpoint }\end{array}$ & $\begin{array}{l}\text { Moderately } \\
\text { susceptible } \\
\text { breakpoint }\end{array}$ & $\begin{array}{c}\text { Resistant } \\
\text { breakpoint }\end{array}$ & $\begin{array}{l}\text { MIC range } \\
(\mu \mathrm{g} / \mathrm{mL})\end{array}$ & $\begin{array}{l}\mathrm{MIC}_{50} \\
(\mu \mathrm{g} / \mathrm{mL})\end{array}$ & $\begin{array}{l}\mathrm{MIC}_{90} \\
(\mu \mathrm{g} / \mathrm{mL})\end{array}$ & $\begin{array}{l}\text { Number } \\
(\%) \text { of } \\
\text { susceptible } \\
\text { strains }\end{array}$ & $\begin{array}{l}\text { Number } \\
(\%) \text { of } \\
\text { moderately } \\
\text { susceptible } \\
\text { strains }\end{array}$ & $\begin{array}{l}\text { Number } \\
(\%) \text { of } \\
\text { resistant } \\
\text { strains }\end{array}$ \\
\hline $\begin{array}{l}\text { M. abscessus } \\
\text { subsp. } \\
\text { abscessus } \\
(n=45)\end{array}$ & 14 & $\leq 2$ & 4 & $\geq 8$ & $1->32$ & $>32$ & $>32$ & $5(10 \%)$ & $3(7 \%)$ & $37(83 \%)$ \\
\hline $\begin{array}{l}\text { M. abscessus } \\
\text { subsp. } \\
\text { massiliense } \\
(n=25)\end{array}$ & 14 & $\leq 2$ & 4 & $\geq 8$ & $\leq 0.0625->32$ & 0.25 & 0.25 & $24(96 \%)$ & $0(0 \%)$ & $1(4 \%)$ \\
\hline $\begin{array}{l}\text { M. fortuitum } \\
(n=23)\end{array}$ & 3 & $\leq 2$ & 4 & $\geq 8$ & $\leq 0.0625->32$ & 0.5 & 4 & $21(92 \%)$ & $1(4 \%)$ & $1(4 \%)$ \\
\hline $\begin{array}{l}\text { MAC } \\
(n=131)\end{array}$ & 7 & $\leq 8$ & 16 & $\geq 32$ & $\leq 0.0625->32$ & 0.5 & 8 & 124 (95\%) & $1(1 \%)$ & $6(4 \%)$ \\
\hline $\begin{array}{l}\text { M. kansasii } \\
(n=34)\end{array}$ & 7 & $\leq 16$ & - & $>16$ & $\leq 0.0625-16$ & $\leq 0.0625$ & 1 & $34(100 \%)$ & - & $0(0 \%)$ \\
\hline
\end{tabular}

TABLE 4: Clarithromycin susceptibility testing result of $M$. chelonei and M. fuerth.

\begin{tabular}{lcccc}
\hline Species & $\begin{array}{c}\text { Incubation } \\
\text { days }\end{array}$ & $\begin{array}{c}\text { Isolate's } \\
\text { number }\end{array}$ & MIC value & DST result \\
\hline & & 2 & $\leq 0.0625$ & Susceptible \\
M. chelonei & 3 & 3 & $\leq 0.0625$ & Susceptible \\
$(n=5)$ & & 13 & $\leq 0.0625$ & Susceptible \\
& & 17 & 0.125 & Susceptible \\
& & $\leq 0.0625$ & Susceptible \\
$\begin{array}{l}\text { M. fuerth } \\
(n=2)\end{array}$ & 3 & 12 & 0.25 & Susceptible \\
\hline
\end{tabular}

and others susceptible. In Table 3, it shows that most $M$. abscessus subsp. massiliense (96\%) strains were susceptible to clarithromycin, and the resistant proportion was low (4\%). However, the other subspecies, M. abscessus subsp. abscessus, had an entirely different result. Most of M. abscessus subsp. abscessus (83\%) strains were resistant to clarithromycin, and there were only $10 \%$ susceptible to it. As SGM, MAC had the biggest proportion of isolates, and the number was 131 isolates. Similarly, most MAC (95\%) were susceptible to clarithromycin, and the resistant proportion was low (4\%). M. Kansasii had the highest susceptible proportion. All of them (100\%) were susceptible to clarithromycin. The sample size of $M$. chelonei and $M$. fuerth was small, so it is inappropriate to calculate the $\mathrm{MIC}_{50}, \mathrm{MIC}_{90}$, and proportions of susceptible, moderately susceptible, and resistant strains. Table 4 displays each isolate's MIC value of M. chelonei and M. fuerth. All of them were susceptible to clarithromycin.
3.3. Clarithromycin Resistant Mechanism. erm(41) genes in subspecies of $M$. abscessus showed characteristic differences. Compared with its homologues in M. abscessus subsp. abscessus, the erm(41) in M. abscessus subsp. massiliense maybe dysfunctional due to deletion of nucleotides. For 49 isolates, the erm(41) gene segment was sequenced successfully. Nine isolates belonged to M. abscessus subsp. massiliense, and all the $\operatorname{erm}(41)$ of which were incomplete. The rest 40 isolates belonged to M. abscessus subsp. abscessus, and all the erm(41) of which were complete. After analyzing these erm(41) gene segments, it showed that (Table 5) except $1 M$. abscessus subsp. abscessus isolate which had C28 in erm(41), nearly all the $M$. abscessus subsp. abscessus strains resistant to clarithromycin had T28 in erm(41). However, all the M. abscessus subsp. abscessus strains susceptible to clarithromycin had C28 in erm(41). It shows that erm(41) sequence differentiated these two subspecies, and its specific features were predictive for clarithromycin susceptibility or resistance.

\section{Discussion}

4.1. Species Identification. The proportions of different species are variable, according to geographical distribution. Geographical variation occurs in the pattern of NTM isolation across different countries. $M$. fortuitum was the commonest species reported from Iran and Turkey whereas MAC predominated most of the European countries and Brazil. In Korea, M. abscessus is the second most common pathogen responsible for lung disease caused by NTM, following the MAC [7]. This is the first study to focus primarily on the clinical relevance of the species 
TABLE 5: The relationship between clarithromycin's resistance and erm(41).

\begin{tabular}{lcccc}
\hline Subspecies & $\begin{array}{c}\text { MIC value of day } 14 \\
(\mu \mathrm{g} / \mathrm{mL})\end{array}$ & Number & DST result & The base at size 28 of erm(41) \\
\hline $\begin{array}{l}\text { M. abscessus subsp. abscessus } \\
(n=40)\end{array}$ & $>32$ & $n=34$ & $\mathrm{R}$ & $\mathrm{T}$ \\
& 8 & $n=1$ & $\mathrm{R}$ & $\mathrm{C}$ \\
$\mathrm{P}$ & $1-4$ & $n=5$ & $\mathrm{~S}$ & $\mathrm{C}$ \\
\hline
\end{tabular}

differentiation among NTM in Beijing, and the largest study of its kind, with almost 278 patients' sputum samples included. In this test, SGM had the larger proportion of NTM than RGM. Among SGM, MAC had the largest proportion in NTM. Nearly half of NTM belonged to MAC. And the second-largest proportion of NTM was $M$. abscessus. Nearly a quarter of NTM belonged to $M$. abscessus. This result is similar to Korea's [8]. M. chelonei, M. fuerth, and M. gordon's proportion was small. There were lots of special species, including $M$. chelonei, M. fuerth, and M. gordon, which had only few amounts, so it is difficult to calculate and analyze the characteristics of these rare species in vitro. In the future we should collect more samples to assess these special species in order to guide clinical treatment.

Two new M. abscessus-related species, M. abscessus subsp. massiliense and M. abscessus subsp. bolletii, were identified, which were previously grouped with $M$. abscessus [5]. Among 40 patients monitored at the National Institutes of Health (Bethesda, MD), the prevalence of M. abscessus subsp. massiliense and M. abscessus subsp. bolletii was $28 \%$ and $5 \%$, respectively [9]. In the Netherlands, $21 \%$ of 39 clinical isolates of $M$. abscessus were identified as M. abscessus subsp. massiliense and 15\% were M. abscessus subsp. bolletii [10]. In France, M. abscessus subsp. massiliense and M. abscessus subsp. bolletii accounted for 22 and $18 \%$ of 50 patients with cystic fibrosis infected with $M$. abscessus, respectively [11]. In Korea, M. abscessus subsp. abscessus and M. abscessus subsp. massiliense are isolated in almost equal numbers among $M$. abscessus infections, whereas $M$. abscessus subsp. bolletii is rare. A previous study showed that nearly half (47\%) of all M. abscessus clinical isolates were identified as M. abscessus subsp. massiliense, although the prevalence of $M$. abscessus subsp. bolletii was low (2\%) [5]. Our test has the same result as Korea's. In this test, more than half $M$. abscessus belonged to $M$. abscessus subsp. abscessus and a little less than a half belonged to M. abscessus subsp. massiliense. There was no one $M$. abscessus subsp. bolletii observed in our test, which means that $M$. abscessus subsp. bolletii accounts for a very small proportion of $M$. abscessus. Differentiation among M. abscessus subsp. abscessus, M. abscessus subsp. massiliense, and M. abscessus subsp. bolletii is challenging for clinical microbiology laboratories. Recent work proved the inaccuracy of single-target sequencing for separating these three taxa within the $M$. abscessus group. In some strains, additional housekeeping genes were analyzed because of the discordant results between $r p o B$ and $h s p 65$ genes analysis $[5,12]$. Sequencing of more other targets (such as $\sec A 1$, soda, and ITS), combined with phylogenomic analysis, has been shown to increase identification accuracy among these different taxa [8]. More recently, a multilocus sequence analysis targeting 8 housekeeping genes and a multispacer sequence analysis were reported $[13,14]$. However, all of these methods require genomic sequencing, which is relatively costly and time consuming and may not be available in all clinical microbiology laboratories.

4.2. Drug Susceptibility Testing. NTM infection is a refractory disease that, unlike tuberculosis, does not respond well to conventional antituberculosis drug. With the progress of mycobacterial taxonomy and speciation, it appeared that antibiotic susceptibility of NTM is tightly related to mycobacterial species and, conversely, that a homogenous intrinsic susceptibility pattern may reflect the homogeneity of the species [2,7]. Since many new species have been described recently with the assistance of molecular biology and genomics, we need to assess their intrinsic susceptibility patterns species by species. The macrolide clarithromycin is considered a cornerstone in antimicrobial chemotherapy of NTM infections. Clarithromycin is a 14 -membered ring macrolide that binds to the large ribosomal subunit in the vicinity of the peptidyl transferase center and inhibits protein synthesis, which results in the arrest of bacterial growth. Clarithromycin is given orally and is highly active against many species of NTM. It is the only drug of demonstrated efficacy that can be administered orally. In all of the studies so far, most variations were reported in the clarithromycin susceptibility results within different NTM species even with strains precisely identified and even with reference strains $[5,15,16]$. For example, $M$. abscessus subsp. bolletii was shown to be clarithromycin resistant by Adékambi and Drancourt [15] and susceptible by Leao et al. [16]. NTM species show a characteristically different response to clarithromycin from others, and precise identification of these species would be important for the treatment of infected patients. This is why we would like to propose that clarithromycin susceptibility be assessed on a molecular basis for all new cases of M. abscessus group infections and that the outcomes of clarithromycin therapy be observed as follows. In this study, we evaluated clarithromycin's activity against different NTM species, since this is the single drug CLSI recommended in in vitro testing. The CLSI recommends the use of the radiometric BACTEC 460, the broth-based macrodilution, or the broth microdilution method for the testing of NTM susceptibility to clarithromycin, and it requires weeks to complete from the time of sample collection because of the growing rate of organism.

As the most common NTM species, the treatment outcome of MAC pulmonary disease was disappointing before the use of newer macrolide antibiotics such as clarithromycin and azithromycin. Since 1990 introduction of 
newer macrolide antibiotics, treatment of pulmonary MAC disease has improved patient outcome. The ATS and IDSA recommend that a three-drug regimen be utilized for the treatment of MAC infection, including a macrolide (clarithromycin or azithromycin). The macrolides are the only class of drugs for which a correlation has been demonstrated between in vitro susceptibility results and in vivo clinical response in MAC disease (Griffith, 2007). For this reason, the ATS only recommends routine susceptibility testing for clarithromycin, the macrolide most commonly used for treating MAC disease (Griffith, 2007). In this test clarithromycin showed an excellent inhibition activity against MAC. 95\% MAC were susceptible to it. And the resistance proportion was low. Considering the big sample size of 131 isolates, this result again proves clarithromycin's good inhibition activity against MAC. However, ATS guidelines also reported that, in contrast to Mycobacterium tuberculosis, DST maybe of little use for the treatment of MAC infection, although clarithromycin is the only drug for which the results of susceptibility testing should be taken into consideration in the treatment of MAC infection [2]. We need to conduct a prospective clinical study in order to compare efficacy in vivo and susceptibility data in vitro.

As the second-largest NTM species and one of the most notorious causative agents of disease, drug therapy of $M$. abscessus disease is long, costly, resistant to many antibiotics, and often associated with drug-related toxicities, leading to unsatisfactory treatment results. In the 1990s, clarithromycin became the drug of choice for $M$. abscessus infections, and therapeutic successes were reported. Combination therapy of intravenous amikacin with cefoxitin or imipenem and an oral macrolide has been recommended by the ATS/IDSA and many other experts [2]. However, treatment response rates are not satisfactory and optimal therapeutic regimens and treatment durations are not well established. Two new $M$. abscessus-related species, $M$. abscessus subsp. massiliense and M. abscessus subsp. bolletii, were identified, which were previously grouped with $M$. abscessus, so the previous $M$. abscessus now includes $M$. abscessus subsp. abscessus, M. abscessus subsp. massiliense, and M. abscessus subsp. bolletii. But the data regarding in vitro DST results of these new subspecies are limited. It is reported that M. abscessus subsp. abscessus and M. abscessus subsp. bolletii have different clinical characteristics and treatment responses in vivo and have different DST result in vitro $[12,17]$. For instance, an experiment in Japan shows that with $M$. abscessus subsp. abscessus bronchiectasia may occur more frequently than with $M$. abscessus subsp. bolletii in vitro, and the resistant proportion of imipenem against $M$. abscessus subsp. abscessus is lower than M. abscessus subsp. bolletii [17]. An experiment in Korea shows that M. abscessus subsp. abscessus may have a worse radiographic response on HRCT and microbiologic response than $M$. abscessus subsp. bolletii in vitro, and similarly, the resistant proportion of imipenem against $M$. abscessus subsp. abscessus is lower than $M$. abscessus subsp. bolletii [12]. In our test, it shows that M. abscessus subsp. abscessus and M. abscessus subsp. massiliense displayed different susceptibility to clarithromycin. Most of M. abscessus subsp. abscessus strains were resistant to clarithromycin. However, M. abscessus subsp. massiliense's MIC to clarithromycin is small, and nearly all of the isolates were susceptible to it. From a clinical standpoint, our study has some important therapeutic implications. The inducible resistance to clarithromycin of $M$. abscessus subsp. abscessus isolates means that it can be much more difficult to treat M. abscessus subsp. abscessus lung disease. A much longer duration of intravenous antibiotic therapy and more effective oral antibiotics may be needed to improve treatment outcomes in M. abscessus subsp. abscessus lung disease. The low MIC for clarithromycin and the absence of inducible resistance to clarithromycin suggest that $M$. abscessus subsp. massiliense lung disease may be more effectively treated with a clarithromycin-based antibiotic regimen [12].

M. kansasii is the second most common cause of NTM disease in the United States. In our test it is the thirdlargest species, but the sample size is small. In this test, $M$. kansasii showed an excellent susceptibility to clarithromycin. All the M. kansasii isolates were susceptible to it. However, according to ATS and IDSA, routine susceptibility testing of $M$. kansasii isolates is recommended for rifampin only, and the recommended treatment of $M$. kansasii pulmonary disease does not include clarithromycin. But the excellent in vitro activity of clarithromycin against $M$. kansasii suggests that these agents may be useful in retreatment regimens. $M$. fortuitum infections are rare, and they are usually susceptible to multiple oral antimicrobial agents. In this test, $M$. fortuitum showed a good susceptibility to clarithromycin. All of the $M$. fortuitum isolates were susceptible to clarithromycin, except 1 isolate resistant to it. In vitro susceptibility data are limited because of the extreme fastidiousness of the organism [2]. Optimal therapy is not determined, but ATS and IDSA also recommend that multidrug therapies including clarithromycin appear to be more effective than those without clarithromycin. $M$. chelonei and $M$. fuerth are extremely rare species. In our test, isolates of these two species are very few, so Table 4 only shows every isolate's MIC value. In Table 4 , it shows that the MIC value is very low and all the $M$. chelone and $M$. fuerth strains were susceptible to clarithromycin. Although the sample size is small, we may still suppose that clarithromycin may have a good inhibition activity against these two species.

4.3. Clarithromycin Resistant Mechanism. For M. abscessus, one of the most notorious causative agents of disease, the macrolide clarithromycin is considered as cornerstone in antimicrobial chemotherapy of pulmonary $M$. abscessus infection. Paradoxically, however, M. abscessus infections of the lung are often intractable to clarithromycin chemotherapy, although high doses of clarithromycin may lead to clinical improvement in some patients $[2,4]$. The lack of efficacy of clarithromycin is puzzling, as pretreatment isolates are usually reported as susceptible to clarithromycin when CLSI procedures are used $[3,4]$. Intriguingly, Brown et al. reported that the clarithromycin MIC increased for some M. abscessus isolates if the incubations for the susceptibility assays were extended, although these isolates would still be deemed clarithromycin susceptible if an incubation period based on the previous CLSI guidelines was used [4, 18]. Similarly, Nash and colleagues showed that although a strain 
might appear susceptible after 3 days of in vitro incubation [3], due to induction of the synthesis of a methyltransferase [4], the strain was clarithromycin resistant if incubation was extended to 14 days or if the strain was preincubated with clarithromycin. So in 2012, CLSI guideline extends M. abscessus DST incubation time from 3 days to 14 days. Although $M$. abscessus subsp. massiliense remained a high susceptible proportion after this adjustment, the resistant proportion of $M$. abscessus subsp. abscessus increased to a high level. An explanation for the instability of clarithromycin MIC is that M. abscessus might have inducible resistance to clarithromycin. Inducible resistance to clarithromycin has been suggested as an explanation for the lack of efficacy of clarithromycin-based treatments against $M$. abscessus infection [12]. An erythromycin ribosomal methylase gene, erm(41), has been identified in several isolates of M. abscessus and the presence of the gene was associated with inducible resistance to clarithromycin. The induction of resistance was shown to result from increased expression of erm(41) as demonstrated by an increase in erm(41) RNA levels after exposure to clarithromycin. The functionality of the methylase is dependent on the nucleotide at position 28 of the erm(41) gene. Wild type T28 sequevars show inducible clarithromycin resistance, while C28 sequevars do not [4]. In our test, of all the $40 \mathrm{M}$. abscessus subsp. abscessus isolates which were sequenced successfully, 35 isolates were resistant to clarithromycin, and 34 isolates in them had T28 in erm(41). However, all the 5 M. abscessus subsp. abscessus isolates which were susceptible to clarithromycin had C28 in erm(41). This result also highlights the resistant mechanism above, which means that $\operatorname{erm}(41)$ may be of relevance to clarithromycin resistance. There is also 1 isolate which has C28 in erm(41) and still resistant to clarithromycin. Maybe there are some other resistant mechanisms needed to be analyzed. Although the therapeutic implications of erm(41) require further study, it is anticipated that expression of this gene does confer clinically significant resistance to clarithromycin. And still, the clinical impact of inducible resistance remains to be determined.

\section{Conclusions}

In this study, we find that MAC was the most common pathogen of NTM, and the second one was M. abscessus. However, M. chelonei, M. fuerth, and M. gordon were rare. Clarithromycin had a good inhibition activity against all the NTM species except M. abscessus subsp. abscessus. erm(41) genotype which is likely to be a major determinant of clarithromycin resistance of $M$. abscessus.

\section{Conflict of Interests}

The authors declare that there is no conflict of interests regarding the publication of this paper.

\section{Authors' Contribution}

Wenjuan Nie and Hongfei Duan contributed equally to this work.

\section{Acknowledgments}

All the mycobacterial strains used in this project were acquired from the "Beijing Bio-Bank of Clinical Resources on Tuberculosis." The research paper introduced was funded by Beijing Municipal Administration of Hospitals Clinical Medicine Development of Special Funding Support. And the authors thank Liang Qian and Jiang Guanglu of Reference Lab of Beijing Chest Hospital for their excellent help in the test.

\section{References}

[1] M. T. Henry, L. Inamdar, D. O’Riordain, M. Schweiger, and J. P. Watson, "Nontuberculous mycobacteria in non-HIV patients: epidemiology, treatment and response," European Respiratory Journal, vol. 23, no. 5, pp. 741-746, 2004.

[2] D. E. Griffith, T. Aksamit, B. A. Brown-Elliott et al., "An official ATS/IDSA statement: diagnosis, treatment, and prevention of nontuberculous mycobacterial diseases," The American Journal of Respiratory and Critical Care Medicine, vol. 175, no. 4, pp. 367416, 2007.

[3] Clinical and Laboratory Standards Institute, "Susceptibility testing of mycobacteria, norcardiae, and other aerobic actinomycetes; approved standard," CLSI Document M24-A, CLSI, Wayne, Pa, USA, 2003.

[4] K. A. Nash, A. B. Brown-Elliott, and R. J. Wallace Jr., "A novel gene, erm (41), confers inducible macrolide resistance to clinical isolates of Mycobacterium abscessus but is absent from Mycobacterium chelonae," Antimicrobial Agents and Chemotherapy, vol. 53, no. 4, pp. 1367-1376, 2009.

[5] H.-Y. Kim, Y. Kook, Y.-J. Yun et al., "Proportions of Mycobacterium massiliense and Mycobacterium bolletii strains among Korean Mycobacterium chelonae-Mycobacterium abscessus group isolates," Journal of Clinical Microbiology, vol. 46, no. 10, pp. 3384-3390, 2008.

[6] W. Nie, H. Duan, H. Huang et al., "Species identification using $r p o B$ and $h s p 65$ and susceptibility testing to eight antibiotics of Mycobacterium abscessus subsp. abscessus and Mycobacterium abscessus subsp. bolletii," International Journal of Infectious Diseases, vol. 25, no. 8, pp. 170-174, 2014.

[7] I. Ahmed, K. Jabeen, and R. Hasan, "Identification of nontuberculous mycobacteria isolated from clinical specimens at a tertiary care hospital: a cross-sectional study," BMC Infectious Diseases, vol. 13, article 493, 2013.

[8] W.-J. Koh, O. J. Kwon, K. Jeon et al., "Clinical significance of nontuberculous mycobacteria isolated from respiratory specimens in Korea," Chest, vol. 129, no. 2, pp. 341-348, 2006.

[9] A. M. Zelazny, J. M. Root, Y. R. Shea et al., "Cohort study of molecular identification and typing of Mycobacterium abscessus, Mycobacterium massiliense, and Mycobacterium bolletii," Journal of Clinical Microbiology, vol. 47, no. 7, pp. 1985-1995, 2009.

[10] J. van Ingen, R. de Zwaan, R. P. N. Dekhuijzen, M. J. Boeree, and D. van Soolingen, "Clinical relevance of Mycobacterium chelonae-abscessus group isolation in 95 patients," Journal of Infection, vol. 59, no. 5, pp. 324-331, 2009.

[11] A.-L. Roux, E. Catherinot, F. Ripoll et al., "Multicenter study of prevalence of nontuberculous mycobacteria in patients with cystic fibrosis in France," Journal of Clinical Microbiology, vol. 47, no. 12, pp. 4124-4128, 2009. 
[12] T. Harada, Y. Akiyama, A. Kurashima et al., "Clinical and microbiological differences between M. abscessus and Mycobacterium massiliense lung diseases," Journal of Clinical Microbiology, vol. 50, no. 11, pp. 3556-3561, 2012.

[13] E. Macheras, A. L. Roux, S. Bastian et al., "Multilocus sequence analysis and $r p o B$ sequencing of Mycobacterium abscessus strains," Journal of Clinical Microbiology, vol. 49, no. 2, pp. 491499, 2011.

[14] M. Sassi, I. Ben Kahla, and M. Drancourt, "Mycobacterium abscessus multispacer sequence typing," BMC Microbiology, vol. 13, article 3, 2013.

[15] T. Adékambi and M. Drancourt, "Mycobacterium bolletii respiratory infections," Emerging Infectious Diseases, vol. 15, no. 2, pp. 302-305, 2009.

[16] S. C. Leao, E. Tortoli, C. Viana-Niero et al., "Characterization of mycobacteria from a major Brazilian outbreak suggests that revision of the taxonomic status of members of the Mycobacterium chelonae-M. abscessus group is needed," Journal of Clinical Microbiology, vol. 47, no. 9, pp. 2691-2698, 2009.

[17] W. J. Koh, K. Jeon, N. Y. Lee et al., "Clinical significance of differentiation of Mycobacterium massiliense from M. abscessus," The American Journal of Respiratory and Critical Care Medicine, vol. 183, no. 3, pp. 405-410, 2011.

[18] B. A. Brown, R. J. Wallace Jr., G. O. Onyi, V. de Rosas, and R. J. Wallace III, "Activities of four macrolides, including clarithromycin, against Mycobacterium fortuitum, Mycobacterium chelonae, and M. chelonae-like organisms," Antimicrobial Agents and Chemotherapy, vol. 36, no. 1, pp. 180-184, 1992. 


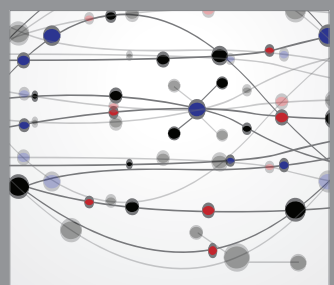

The Scientific World Journal
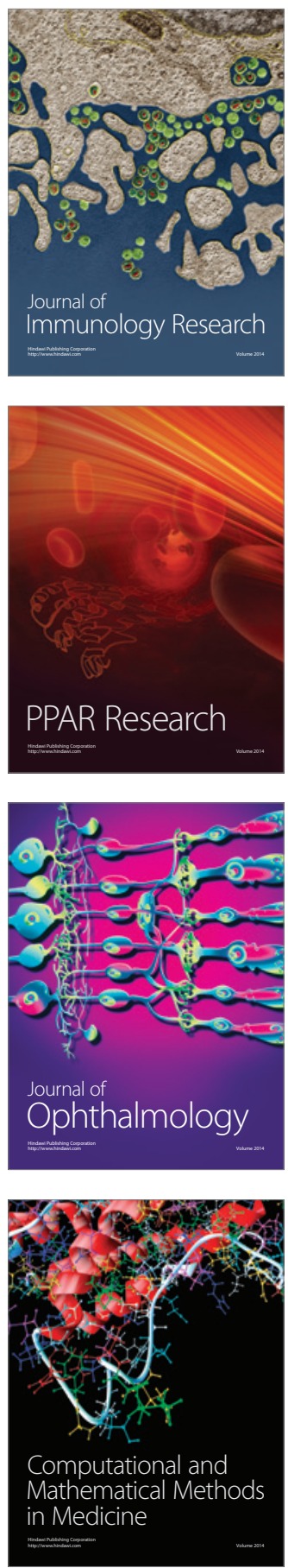

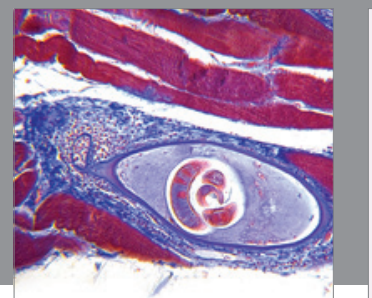

Gastroenterology

Research and Practice
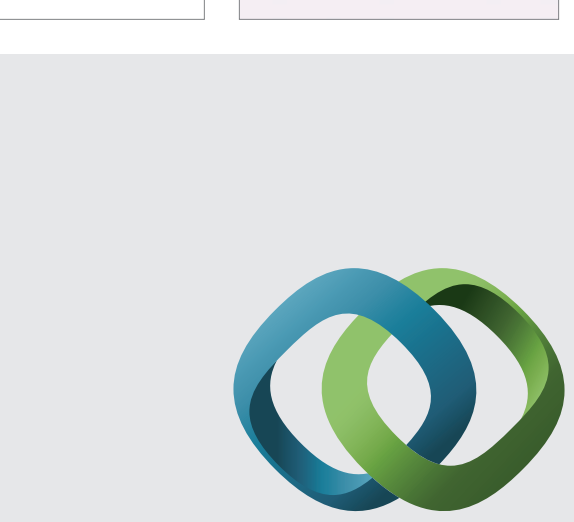

\section{Hindawi}

Submit your manuscripts at

http://www.hindawi.com
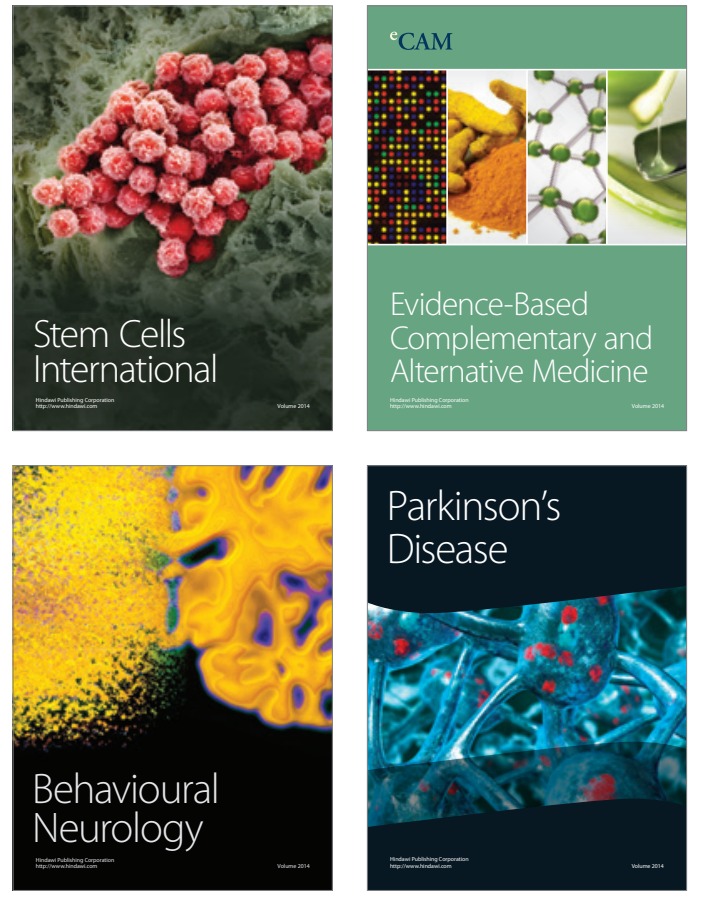
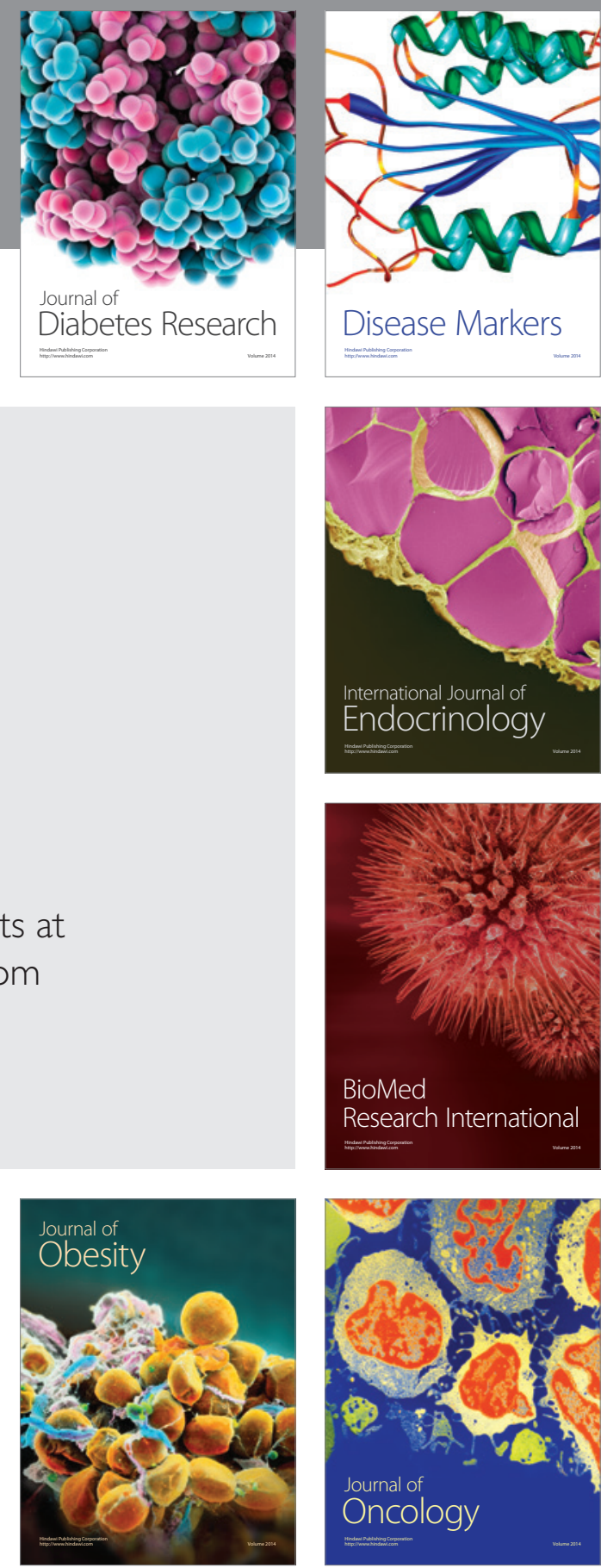

Disease Markers
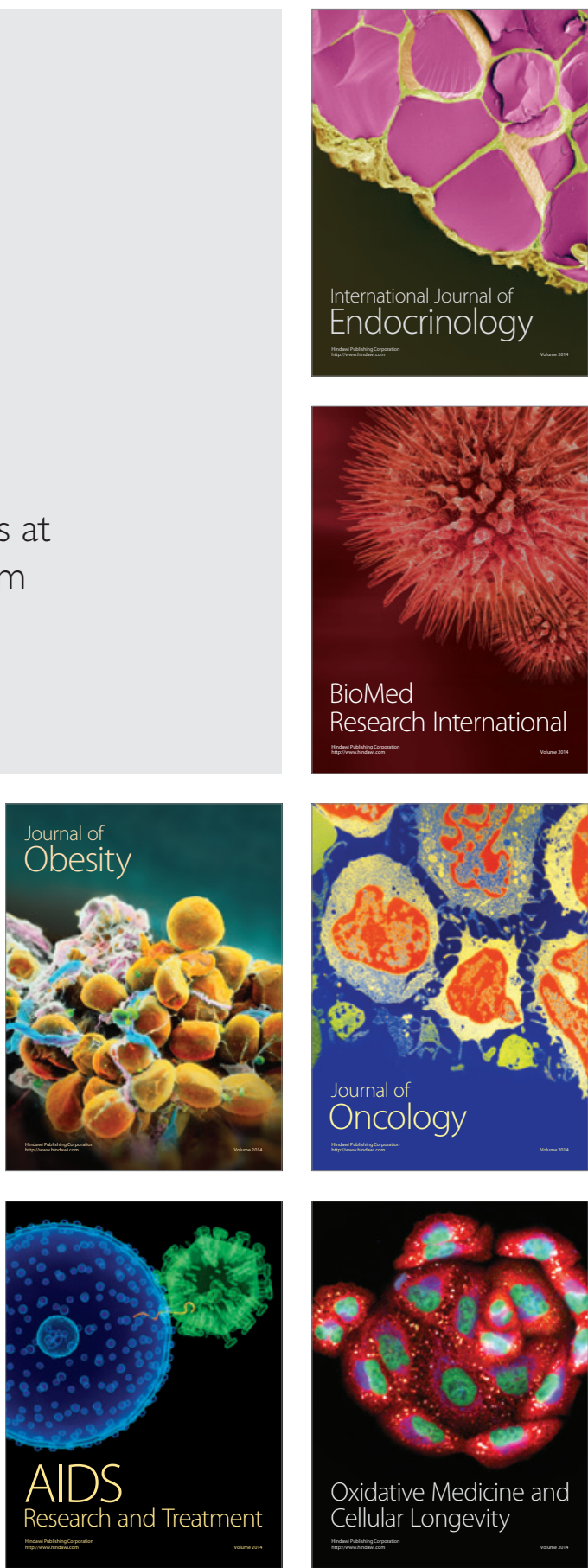